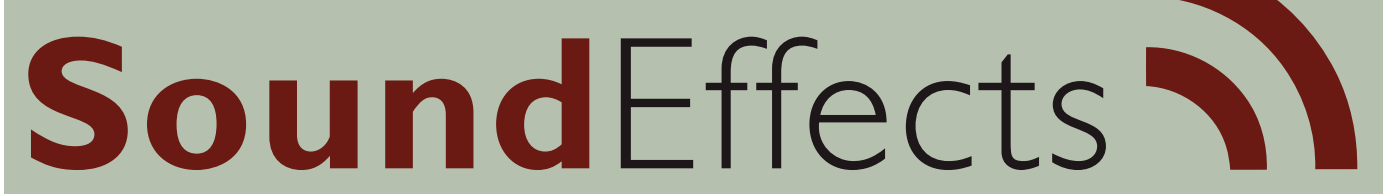

An Interdisciplinary Journal of Sound and Sound Experience

\title{
Vadim Keylin
}

\section{Jordan Lacey: Sonic Rupture}

A Practice-led Approach to Urban Soundscape Design,

Bloomsbury, 2016 
When R. Murray Schafer started the acoustic ecology project in the 1970s, he had a very pragmatic goal in mind: to make the contemporary cities more liveable. The documentation and study of soundscapes was intended as a way to develop a discipline of acoustic design, a way to transform the harsh sonorities of the urban environment into something more welcoming and friendly. Alas, these intentions never led to a tangible practice - until, it seems, now. In his book Sonic Rupture: A Practiceled Approach to Urban Soundscape Design the Australian sound artist Jordan Lacey offers exactly what Schafer hoped for: actionable insights into how cities could be made hospitable with sound.

Unsurprisingly, much of the book is written in dialogue with acoustic ecology. Sonic Rupture's very foreword is titled 'A Note to Acoustic Ecologists' and details the author's complicated relationship with the discipline. Lacey's stated goal is to bring acoustic ecology up-to-date with the recent developments in sound studies, by which he primarily means the sound-focussed branch of speculative realism.

The book's critique of acoustic ecology unfolds along two primary axes. First, Lacey takes issue with the discipline's phenomenological leanings, its emphasis on individual perception, ignoring the interconnected existence of living and non-living bodies. As an alternative he turns to the affect theory, particularly its sound studies version in Steve Goodman's concept of the 'sonic affect'. Rather than locating sound within a person's listening, it is reimagined as a pre-personal continuum that immerses numerous bodies, affecting their experiences. While a challenge to the hegemony of phenomenological approaches in sound studies is long overdue, it bears a question of whether the equally ubiquitous speculative realisms are a better alternative. However, within the context of Sonic Rupture this seems more of a tactical alliance formed to achieve a simpler, but nevertheless important goal. Namely, the affect theory allows Lacey to rethink the role and possible applications of urban noise as an artistic material rather than something to be avoided at all costs.

This feeds into the second line of questioning, which concerns the aestheticising of the soundscape. Acoustic ecologists have a strong and, in Lacey's view, rather unfounded preference for the natural and rural soundscapes over urban ones. They assign different values to sounds depending on where they come from rather than what effect they have - a fundamentally impossible operation within the affect theory framework of Sonic Rupture.

A critique of the very concept of nature aside, for Lacey, the problem with urban noise lies not with its intrinsic qualities, be it its origin, its lo-fi quality or its volume level. Rather, it is the ubiquity and uniformness that are detrimental to the soundscape and the well-being of the city's inhabitants. And even at that, noise is not the enemy, but a symptom of a greater problem that Lacey dubs 'functionalist imperatives' - 'functions, that have become imperative to urban life' (10). These functions subject urban life into uniform and repetitive rhythms, stifling creativity. They are 
the reason behind most homogenous and monotone parts of the urban soundscape - the noises produced by traffic and air conditioning units. Lacey, however, opts to use the noise's ability to disrupt the status quo to turn it on the functionalist imperatives and create 'sites of respite' (73).

Sonic Rupture thus presents an important step in the evolution of approaches to urban noise. Lacey bridges the ecological thinking of the World Soundscape Project with the artistic appreciation for the unwanted sounds found in for example the works of John Cage. Brought together, these concepts offer an effective - and affective - tool to take the public spaces back from the consumerist narratives. Nevertheless, it is hard not to notice that Lacey's argument is also rather aesthetics-based. Where acoustic ecologists assessed the value of a sonic environment along the natu$\mathrm{ral} / \mathrm{unn}$ atural axis, he does the same thing with the diverse/homogenous one.

However, the most important difference between Lacey's approach and that of acoustic ecology is telegraphed by the book's subtitle: It is practice-led. It is hard to overemphasise how this practical grounding affects the material of the book. In Sonic Rupture's lengthiest third chapter Lacey details his journey as a sound artist from following the acoustic ecology imperatives to a new understanding of soundscape design. A detailed account of several artistic projects, all realised in different parts of Melbourne, relays the story of the author's shifting attitudes: from a search for noise-free 'sites of respite' to embracing noise as a means of creating such sites. This journey takes the author through five principal stages that represent five principal strategies in the Sonic Rupture model: subtraction, addition, transformation, passion and disclosure. The list, while not exhaustive, serves as a great starting point for the discussion of what can actually be done with urban soundscape rather than what is wrong with it. Lacey relays his thoughts on the issue in the book's final chapter, which details the Sonic Rupture model, while also providing necessary arthistorical context. Both Lacey's personal account and this historical grounding are crucial to understand where the theoretical claims of the book are coming from.

That Sonic Rupture is grounded in the author's creative practice defines both the book's strengths and its weaknesses. Its principal strength lies in the tangible, down-in-the-trenches quality of Lacey's approach to urban noise, making it more than just a scholarly account. Sonic Rupture doubles as a sort of manual on acoustic design of public spaces. In describing his projects and approaches, Lacey spares no technical or organisational detail, providing easily applicable strategies to battle the noise problem in an innovative way.

Unfortunately, the opening theoretical chapters of the book are considerably weaker. While the scope of his sources is comprehensive, the way Lacey cherrypicks parts of them to build his own theoretical framework may seem rather arbitrary. Even more annoying to an academic reader may be the author's refusal to engage in philosophical discussion on the grounds of the book's scope not permit- 
ting it. To put it bluntly, at 178 pages Sonic Rupture is not a terribly overblown text and could benefit from a more thorough explanation of how its theoretical claims came into being.

In a way, the theoretical chapters of the book read as an extended artist statement. To whatever extent Lacey chooses to engage with his theoretical sources is determined and implicitly justified by his creative practice. At times, his argument reads more poetic than scholarly - but then again, this is a critique that can be addressed to many of Lacey's sources themselves. ${ }^{1}$ In the introduction to Sonic Rupture Lacey suggests that the practice-documenting chapters of the book can be read separately or ahead of the theoretical ones. It may be advisable to follow this suggestion, as the later chapters provide some much needed context for the book's daring theoretical claims. Offering valuable insights and innovative approaches to soundscape studies and design, the book certainly deserves to be read with an open mind.

\section{Notes}

1 As Brian Kane does in his article "Sound studies without auditory culture: a critique of the ontological turn” (2015). Sound Studies, 1(1), 2-21. 